Enhancement of ultrasonic surface waves at wedge tips and angled defects

\title{
Enhancement of ultrasonic surface waves at wedge tips and angled defects
}

R.S. Edwards, ${ }^{\text {a) }}$ B. Dutton, A.R. Clough, and M.H. Rosli

University of Warwick, Coventry, CV4 7AL, UK

The behaviour of sound waves interacting with wedges has attracted interest from researchers in geophysics and non-destructive testing. We consider here the nearfield behaviour of Rayleigh waves incident on wedges and surface-breaking defects which propagate at an angle to the surface, such as rolling contact fatigue on rails. It has been shown that, for a detection point on the edge of the crack tip, a very large signal enhancement is observed for shallow angles. We explain this behaviour through considering the effect of the defect geometry, with changes in the frequency.thickness product leading to mode-conversion of the incident Rayleigh wave.

PACS numbers: $43.20+\mathrm{g}$, 43.55.Ka, 81.70.Fy

Keywords: Rayleigh wave, Enhancement, Laser Ultrasound, Ultrasound Modeling

a) Electronic mail: rs.edwards $\left(a_{\text {warwick.aculd }}\right.$ 
Enhancement of ultrasonic surface waves at wedge tips and angled defects

The interaction of sound waves with wedges has applications to several areas of physics, including non-destructive testing (NDT), specifically the interaction of ultrasound with defects with wedge-type geometry ${ }^{1,2}$, and geophysics, e.g. seismic waves incident on ocean wedges $^{3-5}$. The scattered field behaviour has been studied analytically, through experiments, and models, with reports concentrating on the far-field behaviour of reflected Rayleigh and bulk waves. However, to understand the behaviour in the near-field one must consider the geometry of the sample and the wavemodes which will propagate near the tip.

In NDT, wedges have been studied to probe the properties of dispersive flexural wedge waves $^{6,7}$ or as a simplified model of an angled surface-breaking defect ${ }^{2}$; rolling contact fatigue on rails propagates at an angle of around $25^{\circ}$ to the sample surface, and the branching of stress corrosion cracking in pipes could lead to wedge-type defect regions. Rayleigh waves are a good probe of surface-breaking defects ${ }^{8,9}$ and the far-field transmission coefficient varies with depth and angle to the surface ${ }^{10,11}$.

In the near-field, enhancement of the Rayleigh wave in the vicinity of a surface-breaking defect has drawn a lot of recent interest ${ }^{12-17}$. To quantify this enhancement the signal amplitude in a windowed region around the Rayleigh wave arrival time is measured, and the maximum amplitude compared to that of the incident Rayleigh wave. For a detection point close to a slot machined normal to the surface, enhancement has been shown to be due to constructive interference of the incident Rayleigh wave with reflected and mode-converted waves. For angled defects, it has been shown that the enhancement has a large angle dependence, with enhancements of over 40 times the incident signal observed in finite element simulations for the out-of-plane displacement for shallow angles ${ }^{12}$. This large enhancement will have implications to 'fingerprinting' the presence of a defect in NDT, while the very large out-of-plane tip displacement may need to be considered for earthquakes near ocean wedges. In this paper we consider the near-field behaviour for Rayleigh waves incident on wedges or angled surface-breaking defects, and use consideration of the changing frequency·thickness product to explain the signal enhancement.

The behaviour of ultrasound in the near- and far-field of angled defects and wedges has been investigated using laser ultrasound experiments and finite element method (FEM) models. Laser ultrasound gives many benefits over conventional contact measurements, such as the non-contact nature, high spatial resolution and generation of broadband signals ${ }^{18}$. A pulsed Nd:YAG laser (1064 nm wavelength, $10 \mathrm{~ns}$ pulse duration) was used for generation 
Enhancement of ultrasonic surface waves at wedge tips and angled defects

in the thermoelastic regime, focussed into a line source of approximate dimensions $6 \mathrm{~mm}$ by $100 \mu \mathrm{m}$. Detection used a two-wave mixer laser interferometer, sensitive to the out-ofplane component of the surface displacement, with a bandwidth of $125 \mathrm{MHz}^{19}$. Aluminium samples were produced containing machined slots with a range of angles to the surface ${ }^{10}$, plus a wedge of apex angle $10^{\circ}$. FEM models used PZFlex, with a loading force applied on the sample surface in the form of a dipole ${ }^{10}$.

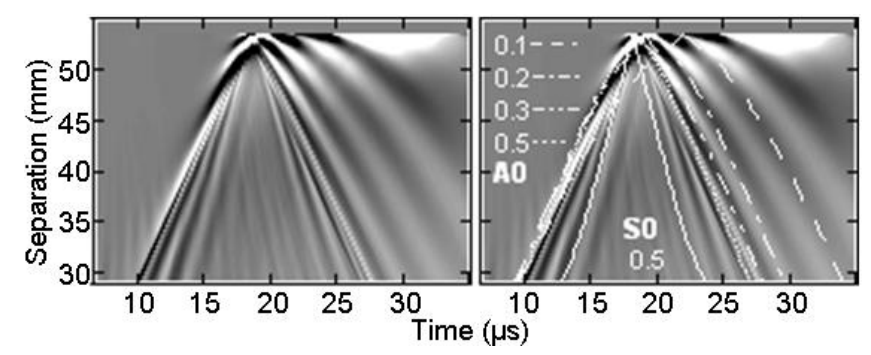

FIG. 1. (a) FEM results for $10^{\circ}$ wedge sample; (b) same, with calculated arrival times of different frequencies from eqn. 2.

To image the behaviour of Rayleigh waves incident on a wedge B-scans have been produced from experimental and model data, with signal amplitude shown by the greyscale (a near-field example is shown in fig. 1). The far-field behaviour of seismic waves scattered from ocean wedge tips has been studied extensively in references ${ }^{3-5,20}$. These papers consider an incident Rayleigh wave which is reflected and transmitted at the tip and/or mode converted into shear and longitudinal wavemodes propagating into the bulk of the wedge. Cooper ${ }^{20}$ recently revisited this problem with modelling of multiple reflections of bulk waves near the tip. The expected reflections of longitudinal and shear wavemodes are observed in our data in the far-field. However, in the near field (fig. 1) these modes disappear and the shape of the incident Rayleigh-pulse becomes more complex.

A wedge or angled defect cannot be treated simply as a quarter-space, but the variation in thickness and its effect on the wavemodes propagating must be taken into account (see eg. refs. ${ }^{21-24}$ for the behaviour of Lamb waves in tapered waveguides or plates with gradual changes in thickness). In a wedge, $f \cdot d$, where $f$ is the wave frequency and $d$ the sample thickness, will be small near the tip. The phase velocity of the waves $\left(c_{p}\right)$ can be calculated analytically, following Rose and other authors ${ }^{25}$, with the group velocity $c_{g}$ calculated using

$$
c_{g}=c_{p}^{2}\left[c_{p}-(f d) \frac{d c_{p}}{d(f d)}\right]^{-1}
$$


Enhancement of ultrasonic surface waves at wedge tips and angled defects

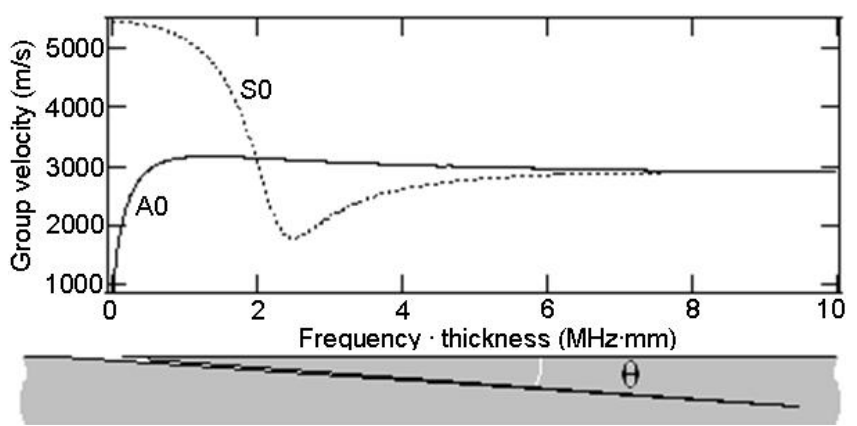

FIG. 2. Group velocity of the two fundamental modes (A0 and $\mathrm{S} 0$ ) as a function of frequency·thickness. $f \cdot d$ will change with position for waves incident on a wedge.

Group velocity for the two fundamental Lamb-wave modes is shown in fig. 2 along with a schematic of an angled defect, showing the change in $f \cdot d$ and hence wave velocity as a surface wave propagates into a wedge.

During propagation, as $f \cdot d$ reduces, the Rayleigh wave will become more Lamb-wave-like as wave energy reaches the far side of the wedge/defect ${ }^{26}$. This is shown in fig. $3(\mathrm{a}) \&(\mathrm{~b})$ for two snapshots of the out-of-plane displacement of an incident surface wave as it approaches the tip for the $10^{\circ}$ wedge FEM model. In (a) the surface wave is Rayleigh-like. However, as the wave propagates further (shown in (b)) some wave energy reaches the far side of the sample, and the wave can be seen as symmetric and antisymmetric modes with different velocities.

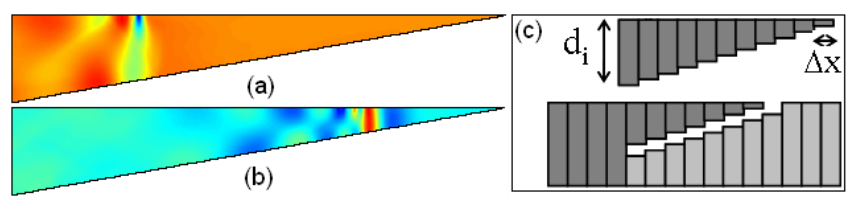

FIG. 3. FEM wedge model: (a) incident Rayleigh-like wave; (b) the spread of the wave to both faces. (c) Splitting the sample into sections.

The arrival time of the surface waves can be calculated by splitting the wedge or defect into $N$ sections, each of width $\Delta x$ (shown in fig. 3(c)) with the thickness varying from the maximum thickness to zero ${ }^{21}$. The travel time for a propagation distance of $l=N \Delta x$ is then

$$
t(f)=\int_{0}^{l} \frac{1}{c_{g}(f, d(x))} d x \approx \sum_{0}^{N} \frac{\Delta x}{c_{g}\left(f, d_{i}\right)}
$$


Enhancement of ultrasonic surface waves at wedge tips and angled defects

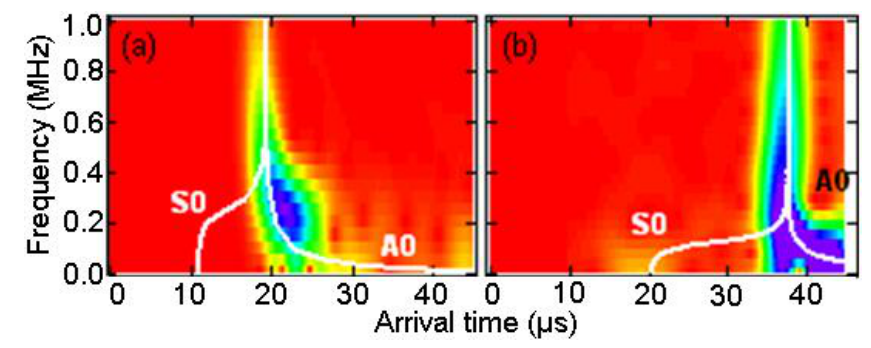

FIG. 4. $10^{\circ}$ wedge; (a) modelled STFT with arrival time calculations as lines, (b) same for experiment.

where $c_{g}\left(f, d_{i}\right)$ is the group velocity for each section thickness calculated from eqn. 1 . The incident waves here are broadband, hence the calculation is repeated for the range of frequencies present in the wave.

Surface ultrasonic waves incident on a $10^{\circ}$ wedge have been modelled, with the resulting Bscan for the near-field shown in fig. 1 for the wedge-tip at $53.6 \mathrm{~mm}$ away from the generation point. As expected, the higher-frequency waves remain Rayleigh-like until close to the wedge tip, whereas the lower frequencies become dispersive. The lines superimposed on the righthand figure show calculated arrival times using eqn. 2 for several frequencies, adapted for both incident and reflected modes. Only one frequency $(0.5 \mathrm{MHz})$ is shown for the $\mathrm{S} 0$ mode; we calculate the out-of-plane surface displacement, and hence we will mainly detect the A0 mode near the tip ${ }^{25}$. For the A0 mode, frequencies from 0.1 to $0.5 \mathrm{MHz}$ are shown; above this frequency the wavemodes have very similar arrival times. Very good agreement is shown with the modelled wave pattern. These calculations also explain the enhancement pattern observed, with its characteristic alternating light and dark regions, which has previously been used to identify angled defects ${ }^{27}$.

The mode-conversion of the incident Rayleigh wave can be further confirmed by the timefrequency behaviour of the enhanced wave at the tip (53.6 mm in fig. 1). Short time Fourier transforms (STFTs) are shown in figure 4 for both (a) model and (b) experimental data on a $10^{\circ}$ wedge. The sensitivity of the out-of-plane detection to primarily the A0 mode is clear ${ }^{25}$. Lines on the figure show the calculated arrival times using eqn. 2, in excellent agreement with the detected signals; note that the model set-up has smaller generator to detector separation due to memory constraints and hence earlier arrival times.

This analysis can be extended to surface-breaking defects which are angled with respect 
Enhancement of ultrasonic surface waves at wedge tips and angled defects

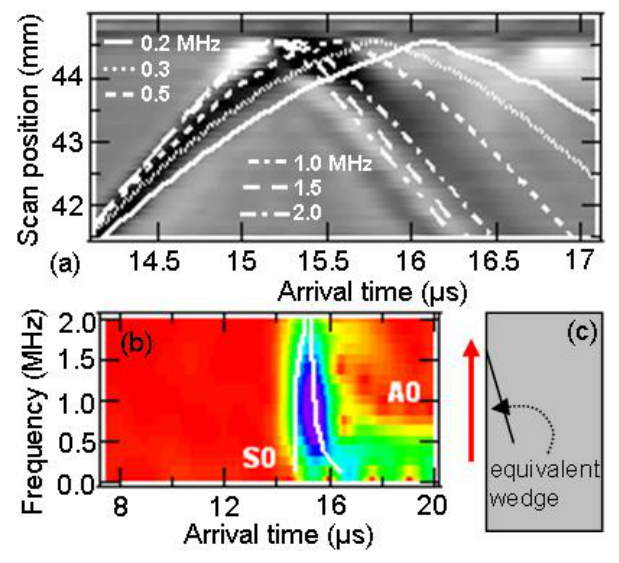

FIG. 5. Experimental results for a $2 \mathrm{~mm}$ long defect inclined at $20^{\circ}$ to the sample surface. (a) B-Scan, (b) STFT of the enhanced wave at the tip, (c) schematic of sample.

to the surface, forming wedge-shaped areas (fig. 5(c)). We show as an example experimental results on a defect oriented at $20^{\circ}$ to the surface, of length $2 \mathrm{~mm}$ and hence having a horizontal extent of $1.88 \mathrm{~mm}$. Fig. 5(a) shows the near-field B-scan, along with arrival time calculations from eqn. 2 for the A0 mode $(0.2,0.3,0.5,1.0,1.5$ and $2.0 \mathrm{MHz})$. Here, a significant proportion of the Rayleigh wave energy will pass underneath the $\operatorname{defect}^{9}$. However, the higher frequencies will see a wedge-like sample. Again, dispersion is clear (also in the STFT of the enhanced wave in fig. 5(b)), and the distinctive enhancement pattern can be explained.

When a Rayleigh wave is incident on a wedge-like defect the changing frequency·thickness leads to a splitting into A0 and S0-like modes. These modes have an arrival time which depends on frequency, and hence for a broadband incident pulse a spreading of the wave is observed. For a defect oriented normal to the sample surface, constructive interference of the incident and reflected Rayleigh waves and the mode converted surface skimming longitudinal leads to a larger signal enhancement in the in-plane than the out-of-plane signal ${ }^{16}$. For a wedge or angled defect the interference of the Lamb-wave-like modes will also lead to signal enhancement, with the focussing of the wave energy into the wedge tip giving a very large enhancement for shallow angles. The magnitude will depend on the component of the wave motion measured (in-plane or out-of-plane), and we must consider the displacement profile of the waves at the surface of the wedge. From reference ${ }^{25}$, the S0 mode tends towards large in-plane and small out-of-plane displacement for low $f \cdot d$, whereas the A0 has a larger 
Enhancement of ultrasonic surface waves at wedge tips and angled defects

out-of-plane displacement. For defect angles close to $90^{\circ}$ there is little spreading in time of the A0 and S0 modes, and hence we would still expect a larger in-plane enhancement when comparing signals to the incident Rayleigh wave. However, for shallower angles, depending on the incident frequency the S0 and A0 modes arrive at different times. Therefore, as the windowed region (set for the Rayleigh arrival time) covers more of the A0-like mode, we would expect a large out-of-plane enhancement for shallow angles. This has been observed experimentally ${ }^{12}$.

In NDT the scanning laser source technique ${ }^{17}$, where an enhancement is seen due to changing generation conditions when the generation laser is above a defect, has been suggested as a method of fingerprinting the presence of a defect. However, very little angle dependence is observed in this enhancement ${ }^{12}$. Therefore, when there is the possibility of angled defects a measurement using a scanned detection system will be more appropriate for identifying and characterising defects.

This work was funded by the European Research Council under grant 202735.

\section{REFERENCES}

${ }^{1}$ BV Budaev and DB Bogy, Wave Motion 22 239-257 (1995)

${ }^{2}$ VM Babich, VA Borovikov, LJ Fradkin, V Kamotski and BA Samokish, NDT \& E International 37(2) 105-109 (2004)

${ }^{3}$ K Fujii, S Takeuchi, Y Okano and M Nakano, B. Seismol. Soc. Am. 74(1) 41-60 (1984)

${ }^{4}$ S Takeuchi, K Fujii and M Nakano, B. Seismol. Soc. Am. 74(6) 1593-1603 (1984)

${ }^{5}$ Z Alterman and R Nathaniel, B. Seismol. Soc. Am. 65(6) 1697-1719 (1975)

${ }^{6} \mathrm{C}-\mathrm{H}$ Yang and K-Y Tsai, Jap. J. of Appl. Phys. 43(7A) 4392-4393 (2004)

${ }^{7}$ X Jia and M de Billy, App. Phy. Lett. 61(25) 2970-2972 (1992)

${ }^{8}$ X Jian, S Dixon, N Guo and RS Edwards, J. of Appl. Phys. 101(6) 064906 (2007)

${ }^{9} \mathrm{RS}$ Edwards, S Dixon and X Jian, Ultrasonics 44(1) 93-98 (2006)

${ }^{10}$ B Dutton, AR Clough, MH Rosli and RS Edwards, NDT\&E International 44(4) 353-360 (2011)

${ }^{11}$ VK Kinra and BQ Vu, J. Acoust. Soc. Am. 79(6) 1688-1692 (1986)

${ }^{12}$ B Dutton, AR Clough and RS Edwards, J. Nondestruct. Eval. 30(2) 64-70 (2011)

${ }^{13}$ JL Blackshire and S Sathish, Appl. Phys. Lett. 80(18) 3442-3444 (2002) 
Enhancement of ultrasonic surface waves at wedge tips and angled defects

${ }^{14}$ S Boonsang and RJ Dewhurst, Appl. Phys. Lett. 82(19) 3348-3350 (2003)

${ }^{15}$ S Dixon, B Cann, DL Carroll, Y Fan and RS Edwards, Nondestruct. Test. Eval. 23(1) 25-34 (2008)

${ }^{16}$ RS Edwards, X Jian, Y Fan and S Dixon, Appl. Phys. Lett. 87(19) 194104 (2005)

${ }^{17}$ AK Kromine, PA Fomitchov, S Krishnaswamy and JD Achenbach, Materials Evaluation 58(2) 173-177 (2000)

${ }^{18}$ AM Aindow, RJ Dewhurst and SB Palmer, Optics Communications 42(2) 116-120 (1982)

${ }^{19}$ MB Klein, GD Bacher, A Grunnet-Jepsen, D Wright and WE Moerner, Optics Communications 162 79-84 (1999)

${ }^{20}$ JK Cooper, DC Lawton and GF Margrave, Geophysics 75(2) T15-T21 (2010)

${ }^{21}$ L De Marchi, N Speciale and E Viola, NDT\&E Interational 43(3) 265-271 (2010)

${ }^{22}$ N Wakatsuki, K Mizutani and K Nagai, Jpn. J. Appl. Phys. 34 2561-2564 (1995)

${ }^{23}$ Y Cho, IEEE Trans. Ultrason. Ferr. 47(3) 591-603 (2000)

${ }^{24} \mathrm{M}$ Ech-Cherif El-Kettani, F Luppea and A Guilleta, Ultrasonics 42(1-9) 807-812 (2004)

${ }^{25}$ JL Rose, Ultrasonic waves in Solid Media, Cambridge University Press (1999)

${ }^{26}$ KhB Tolipov, J. Appl. Mech. Tech. Phy. 51(1) 22-30 (2010)

${ }^{27} \mathrm{MH}$ Rosli, RS Edwards, B Dutton, CG Johnson and PT Cattani, Rev. Prog. QNDE 29A\&B 1593-1600 (2010) 